\title{
Computerised Link Analysis
}

\author{
Yu Zhao \\ Healthcare Ergonomics and Patient Safety Unit \\ Dept. of Human Sciences \\ Loughborough University \\ Y.Zhao3@1boro.ac.uk
}

\author{
Sue Hignett \\ Healthcare Ergonomics and Patient Safety Unit \\ Dept. of Human Sciences \\ Loughborough University \\ S.M.Hignett@lboro.ac.uk
}

\author{
Neil Mansfield \\ Dept. of Human Sciences \\ Loughborough University \\ N.J.Mansfield@lboro.ac.uk
}

\begin{abstract}
Motivation - Link Analysis (LA) is a method to study relationships of elements within a system, and it is also used as a data recording method of Naturalistic Decision Making (NDM), e.g. capturing staff movements. However LA has limitations, such as time-consuming data recording and results generating processes. Research approach - Computerised Link Analysis (CLA) system is being developed and tested by computerising template generating, real-time event recording and data analysing processes. Design - The first version of system has been accomplished and its description is included to demonstrate its versatility. Research limitations - This system is still at an early prototype stage and improvements and tests need to be conducted. Value - CLA is the first system which is designed specially for healthcare domain and has proven to save plenty of time compared to manual method. Take away message - CLA system is able to offer analysts an efficient and effective tool to perform LA.

Keywords

Link Analysis, healthcare, template generating (layout generating), data recording (event recording), result generating, tablet $P C$, touching screen.
\end{abstract}

\section{INTRODUCTION AND PROBLEM STATEMENT}

\section{Naturalistic Decision Making}

NDM is defined as studying 'the way people use experience to make decisions, solve problems, and gain situation awareness' (Klein, 1995). Endsley, Klein, Woods, Smith \& Selcon (1995) describe NDM as a means of attempting 'to understand how people process information and perform within complex systems and to develop ways of applying this knowledge within design and training process'. According to Beach, Chi, Klein, Smith \& Vicente (1997), NDM is concerned with how 'reasonably experienced people actually make judgment and decisions in realistic settings'. Models have been built, such as Recognition-Primed Decision making (Klein, 1989), Image Theory (Beach \& Mitchell, 1987) and Situation Assessment Theory (Noble, 1989). NDM has been applied in a large range of domains, such as aviation, medicine, nuclear power, and command control (Mosier \& Orasanu, 1995).

The term of NDM reminds people of the decision event and moment of choice. However, it is different from traditional decision making. Traditional decision-making approaches aim to identify a method to improve the way people make decisions or to make better choices by 'generating a range of options, identifying evaluation criteria, evaluating each option on criterion, calculating results, and selecting the option with highest score'(Klein, 1997). The purpose of $\mathrm{NDM}$ is to describe what proficient people actually do and what strategies they use to make decisions, solve problems and take actions.

Zsambok and Klein (1997) identified four applications of NDM where the: (1) task and setting involve ill-structured problems, uncertain, dynamic environments, shifting ill-defined or competing goals, action/feedback loops, time pressure, high stakes, multiple players, and organisational goals and norms; (2) subjects are experienced participants; (3) locus of interest includes situation awareness, diagnosis, and plan generation rather than fixating on the moment of choice; (4) purpose of research is to describe the strategies people actually use rather than prescribing what they ought to use.

\section{Background}

Healthcare is the prevention, treatment and management of disease or illness and aims to provide services to population to promote health. Ergonomics in healthcare is often discussed as important for offering better working conditions for clinical staff to perform their job effectively, efficiently and safely. LA, as a considerably useful ergonomic method, 
has been used increasingly in the healthcare domain to study and improve the layout of workspaces, e.g. intensive care units (Hignett \& Lu, 2007), emergency departments (Jones, Hignett \& Benger, 2008), ambulances (Ferriera \& Hignett, 2004), etc. However, healthcare is complex and dynamic, full of uncertainty and variance. For example, although providing treatment to a patient presenting with a particular complaint (e.g. chest pain) might seem to include a number of standardised procedures, it is likely that there will be plenty of variance for each task based on the individual differences of each patient (e.g. age, gender, past medical history), and location of presentation (emergency dept, GP clinic, minor injuries unit, ambulance). In order to respond to these short and non-repetitive variance, LA is also used in healthcare domain as a data (event) recording method of NDM to capture information such as the physical movements of clinical staff during an operation. These data can be reviewed to guide people to understand what these experienced staff do and what strategies they use to provide services for the patients, and they are also used frequently for design and training processes. When LA is applied in a healthcare context, it needs to be more sophisticated, efficient and effective to take account of the complexity of the NDM and non-repetitive nature of tasks, however traditional 'pen and paper' method of LA is not capable to support due to time-consuming manual data recording and results generating processes, etc. With the rapid development of computer technologies, some examples of LA with computers have been developed and documented, but none of them is particularly designed for the healthcare domain. Thus, Computerised Link Analysis (CLA) system is being developed and tested to use touch-screen on a Tablet laptop to provide flexibility for template (layout of workspace) generating and simplify data recording and results generating (tallying and counting the links, and the drawing link diagram) processes. The description of developing CLA system is presented to demonstrate the versatility.

\section{Aims and objectives}

The overall aim of CLA system is to use highly developed computer techniques to develop CLA system, and test it in both laboratory and real environments with a range of users (both non expert users and trained users) on its efficiency and reliability, in order to offer ergonomists an efficient and reliable tool to study the layouts in healthcare domain. The objectives are to: (1) become familiar with LA (its definition, procedures, history and development, advantages and disadvantages, and various applications in different areas) and current relevant researches LA with computers; (2) programme software applications, simplify event recording, add more data (chronological order and duration and direction of link), and achieve real-time data collecting and processing; (3) test the efficiency and accuracy of system to ensure CLA is as accurate as the manual LA method but more efficient; (4) recruit both non-expert and trained users to use CLA to gather feedback to both improve CLA and the user interface; (5) test CLA for real-time data recording and processing in real environments (e.g. operating room and ambulance) to ensure it is able to respond to short and nonrepetitive variance during an operation effectively and effectively.

\section{LITERATURE REVIEW}

LA developed primarily from Flow Analysis which aimed to describe 'the nature and sequence of events in a product or process' and study 'the arrangement and dependencies among elements in system' (Nemeth, 2003). However, Flow Analysis can only be used to examine tasks which are 'repetitive and standardized' (Chapanis, 1959); i.e. it is not able to deal with tasks which include unexpected behaviours. Thus, LA was developed as a method which has capability to evaluate all kinds of systems.

LA is a systematic technique for studying human-human, human-machine and machine-machine interactions and seeking to understand how activities in the system are linked together. It has been applied in a large range of areas and proven to be a very useful tool in Ergonomics to improve the design of products. Thomson (1972) depicts it as ' $a$ technique which provides information needed to produce an acceptable arrangement of men and machines in a system'. In LA, systems are composed of links and elements. Links, as noted by Chapanis (1959), are expressed in physical terms: relative frequencies between elements, including movements of hand, eye or body, communications and some kind of abstract relationships, e.g. connections between criminals (Harper \& Harris, 1975) or relationships among measles cases (Stuster \& Chovil, 1994). A spatial link diagram is provided as a product of LA which illustrates all the elements and links presented in an operation. The purpose of LA is to minimise the overall distance of links travelled within the task by rearranging elements, in order to generate an optimal layout to increase efficiency and achieve better performance.

An example is included to explain the definition and procedures of LA. Figure 1(a) illustrates the original layout of an office, in which desk B is located between desk A and C. For one task, the operator has to travel 5 times between A and $\mathrm{C}, 1$ time between $\mathrm{A}$ and $\mathrm{B}$, and 3 times between $\mathrm{B}$ and $\mathrm{C}$. All the links are recorded and depicted in a link diagram (Figure 1(b)), while Figure 1(c) is an alternative illustration, interaction matrix. In order to cut travel distance and obtain optimal layout of the office corresponding to this particular task, desks are re-located as illustrated in Figure 1(d).

Probably the earliest formal application of LA can be dated back to 1917, when the Gilbreth brothers studied the layout of workshops by evaluating overall distances travelled during the operations (Gilbreth \& Gilbreth, 1917). Since then, LA has become a popular and useful tool in rearranging or redesigning human-machine, machine-machine or humanhuman layouts, and has been used in a large range of areas. Many applications in different areas, as well as variations 
in methods of LA, have been developed and documented. Fitts, Jones \& Milton (1950) studied eye movements among instruments on control panel of aircraft while pilots were performing instrumented landing approaches. Data (eye

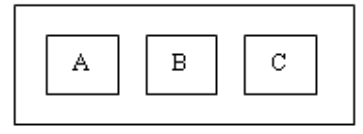

(a) Original layout

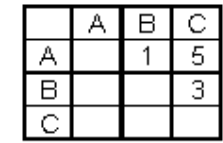

(c) Interaction matrix

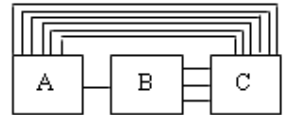

(b) Link diagram

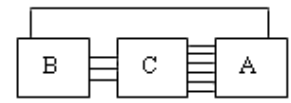

(d) Optimal solution

Figure 1. A LA illustration.

movements) were collected and further analysed leading to a standard arrangement of instruments in the control panel (McCormick, 1970). Chapanis (1959) studied human-human and human-machine layout in the Combat Information Centre (CIC). The path of movements of officers among the complex assemblage of men and machines in CIC was observed during an air attack and data were recorded in order to produce an improved layout. It is likely that Haygood, Teel \& Greening (1964) were the first people to use computers to simplify the method of LA. The C2110M Flight System Checkout Console, used to check out operational equipment in the GAM-77 missile system, was studied. Though the computer used is considerably dated (RECOMP II), they were able to prove that results from computeraided LA were equally accurate and significantly saved time and money. As noted by Lippert (1971), LA has been employed in hospital wards to 'draw a map' which depicts the pattern of a nurse travelling among the patient's beds, utility room and the ward nurse's station. Harper \& Harris (1975) introduced LA into an entirely different field, Police Intelligence, to prevent and control organised crime. The widespread success of LA in criminal investigation encouraged Stuster \& Chovil (1994) to take LA into another field, epidemiology, to map a measles epidemic.

LA has been applied in a wide range of areas and has been proven to be a useful technique to study the relationships among elements in systems. However, as it is being used more frequently, difficulties and problems have started to arise. As noted by Chapanis (1959), LA does not consider the order of links, but simply tally the frequency of each link. Haygood et al. (1964) mentioned that it is possible to analyse tasks which consist of only several hundred steps with pen and paper, however it would be almost impossible when dealing with a task with thousands or more steps. 'The manual process of keeping a tally and counting links becomes prohibitively time-consuming' (Glass, Zaloom \& Gates, 1991). This also leads to the difficulty of drawing an immense schematic link diagram for large system. All these difficulties and problems thus provide a great opportunity for computer facilities (e.g. programming languages, devices such as touch-screen and computer software) to increase the efficiency and improve the performance of LA.

\section{CURRENT ACHIEVEMENTS WITH COMPUTER}

With rapid development of techniques, computers have being applied for a large number of applications. Since 1964, when Haygood et al. succeeded in proving that a computer-based LA could save time and money, researchers have been trying to use computer facilities to improve the performance and simplify the processes of LA.

\section{Computer-Aided Link Analysis (CALA)}

The CALA system designed by Glass et al. (1991) uses Computer-Aided Design (CAD) software to simplify the layout-creating process and capture link distances automatically. During an operation, the observer collects data for LA (links/movements between elements) and tallies the frequency between elements. The CALA system reads in the frequency between elements and link distances (from CAD), as well as other parameters (e.g. element description, wage/cost rate, link criticality value, and link transfer rate etc.), and generates four alternative results. One of them, 'Sorted Link Analysis report', is shown in Figure 2, in which events are listed in descending order of frequency. 


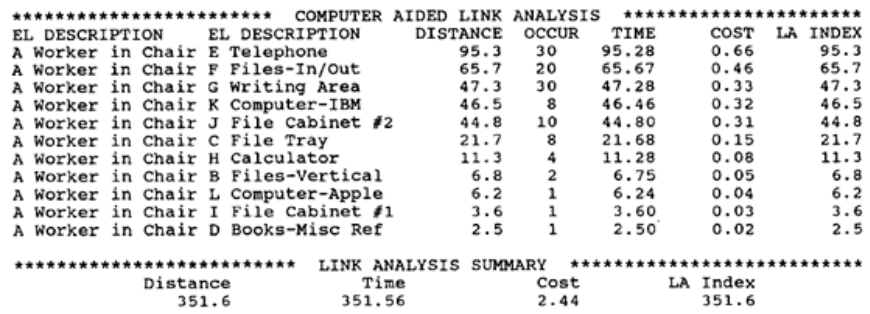

Figure 2. Sorted Link Analysis report. Adapted from Glass et al. (1991).

\section{Advantages and disadvantages of CALA}

Advantages: (1) CAD is employed to simplify layouts creating and link distances capturing; (2) data (both input and output) are stored in ASCII text file, which can be read and used to generate reports outside CALA easily; (3) four alternative results are generated for different situations; (4) optional parameters (e.g. element wage/cost rate, link criticality value, and link transfer rate) are provided.

Disadvantages: (1) the observer records data and tallies and counts the links manually; (2) knowledge of operating the software of CAD is required.

\section{Link Analyzer}

Thorstensson, Axelsson, Morin \& Jenvald (2001) demonstrated an improved method of LA, Extended Link Analysis (ELA), which not only recorded events, but also classified them using a pre-designed category and registered a time stamp when they occurred. Moreover the software 'Link Analyzer' was developed. This software enabled the observer to enter all data (event name, time and classification) into a computer. A study of internal work and communication process in a command post, which enhanced the performance of an emergency operation (e.g. rescue operation), is presented to illustrate its usability. Category of classifications is pre-defined. The layout of the command post is configured using Link Analyzer (Figure 3). The observer monitors and registers a single communication event in the command post by feeding its sender, receiver, and classification (chosen from the category) into Link Analyzer and this is automatically time stamped when the registration occurs. The outcome of Link Analyzer is shown in Figure 4.

Advantages and disadvantages of Link Analyzer

Advantages: (1) Link Analyzer offers useful information such as start/stop time, duration, chronological order and classification of each link; (2) it generates a good structured result, in which events are listed in chronological order.

Disadvantages: (1) only standard classifications which are pre-designed are provided, while variance is not considered; (2) the number of staff members a single observer can monitor is limited; (3) only one communication can be observed at a time, thus it does not support parallel events recording; (4) manual process of entering events into computer is timeconsuming; (5) computer is used mainly as a database (storage tool).

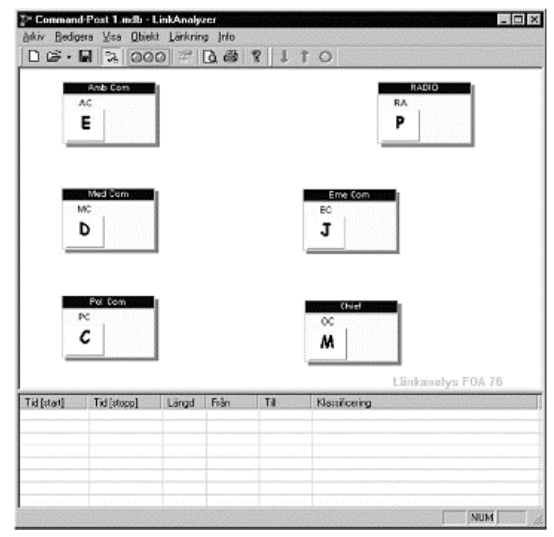


Figure 3. Layout configuration of command post. Adapted from Thorstensson et al. (2001).

Events are registered into table of Link Analyzer below the layout configuration.

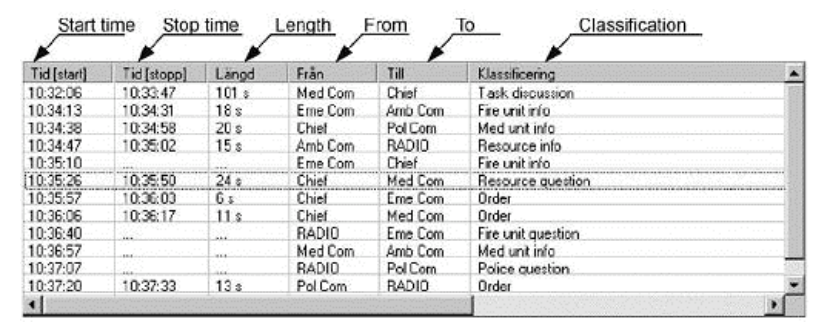

Figure 4. Outcome of Link Analyzer. Adapted from Thorstensson et al. (2001).

Each line represents a event which is registered with 'Start time', 'Stop time', 'Length (time duration)', 'From (sender)', 'To (receiver)' and 'classification (chosen from pre-designed category)'.

\section{The FIT system}

The studies by Held (1999), Held, Bruesch, Krueger \& Pasch, (1999) and Held \& Manser, (2005) are not focused particularly on improving LA, but the by-product is a great achievement of simplifying event recording process, using touch-screen on a mobile hand-held computer device, Personal Digital Assistant (PDA). The system FIT (Flexible Interface Technique) has proven to be helpful and efficient in event recording. 'Flexible interface' means that observer is able to design his/her own interface (layout), using symbols, hand-written texts, sketches, on a paper overlay which covers the touch screen of the PDA. An example of studying interactions among oxpecker birds and a rhinoceros is included and an overlay, with a brief sketch of rhino, is shown in Figure 5. During data recording, the observer registers each event by tapping with a stylus on the related area on the overlay. The co-ordinate and the time of each tapped point are stored in the memory of the PDA. After recording, data are transferred to a computer, where the points are illustrated exactly as they are typed in. The observer encircles the points according to the representations of the overlay by drawing polygons around points (Figure 6). Figure 7 depicts the results of Event-Time Diagram generated by FIT.

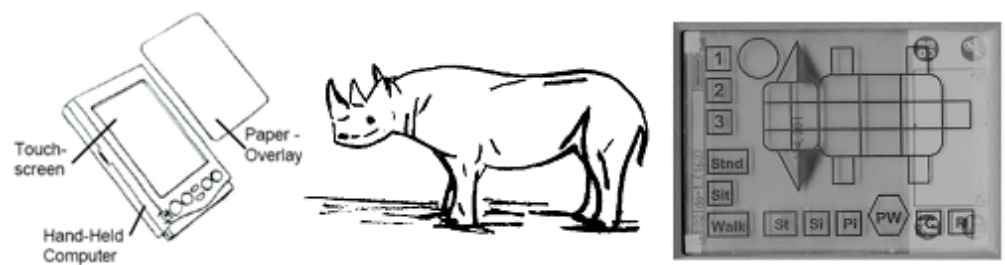

Figure 5. An example of paper overlay, studying behaviours of oxpecker birds and a rhinoceros. Adapted from Held et al. (1999) and Held \& Manser (2005)

Event is recorded by typing the corresponding area on overlay. E.g. when an oxpecker bird lands on the left ear of rhino, left ear of the sketch on overlay is selected using a stylus. Coordinates of this selected point and corresponding time is recorded in memory of PDA.

\section{Advantages and disadvantages of FIT}

Advantages: (1) portable computer device and touch-screen are used to simplify the event recording process; (2) it provides flexibilities in designing layout by creating interface (layout) with pen and paper; (3) direct viewing EventTime List are used as results; (4) useful data, e.g. time stamp, time duration and chronological order of links, are provided; (5) it is easy to operate.

Disadvantages: (1) data are needed to be transferred from PDA to PC and further processed on PC; (2) PDA has limitations, e.g. PDA is not as easily programmable as PC, and memory and size of PDA screen are too small for large system. 


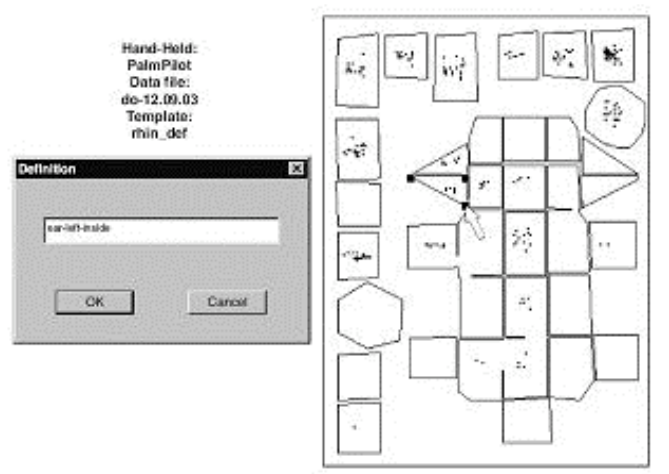

Figure 6. Regroup the points. Adapted from Held \& Manser (2005).

Data (coordinates of typed points and corresponding time) are imported from PDA to PC. Typed points are illustrated in FIT Manager on PC and grouped according to the meaning of overlay (Figure 9).

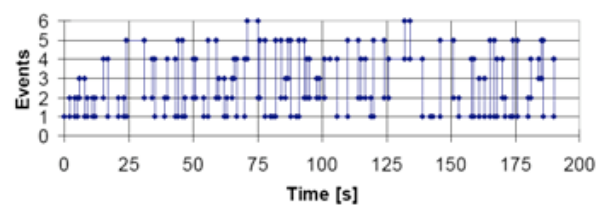

Figure 7. Event-Time Diagram. Adapted from Held \& Manser (2005).

Several current achievements of LA with computer are illustrated, with their advantages and disadvantages. Though there are several limitations within these examples, they provide good ideas of how LA could be improved with a computer and give a future research direction.

\section{SYSTEM DESCRIPTION OF COMPUTERISED LINK ANALYSIS (CLA)}

\section{Description of programmes}

The CLA system uses a Tablet laptop computer with a built-in touch-screen, to increase the efficiency of template generating, data recording and results generating processes of LA and provide analysts with efficient software to study and design workspaces in a healthcare department. According to the different nature of the objectives, the CLA system is divided into two software packages: (1) 'Template Generator' which deals with creating the layouts; (2) 'Data Recorder' which is responsible for recording links on the touch-screen and generating the final results. These two programmes will be discussed respectively.

\section{Template Generator}

The purpose of the Template Generator is to provide flexibility for customising template design. It is designed to offer users a white board on which rectangular box representations of the elements in the system layout can be drawn. As shown in Figure 8, the example system encompasses eight elements (rectangular boxes) which have been created on the white board. Each box is required to be no smaller than $30(\mathrm{px})$ by $30(\mathrm{px})$, otherwise it is difficult to select during the data recording process. If the box is too small, a dialog window will pop up to prompt users to redraw. The boxes which have been created are movable and they can be dragged anywhere inside the white board using mouse. 'Undo' and 'Redo' buttons provide users with an easy way to modify their work, while various colours at the left corner offer decoration options. When the layout is saved, a numbering system assigns each box with a number according to the sequence in which it was created and this number is defined as the name of the box (Figure 8). 


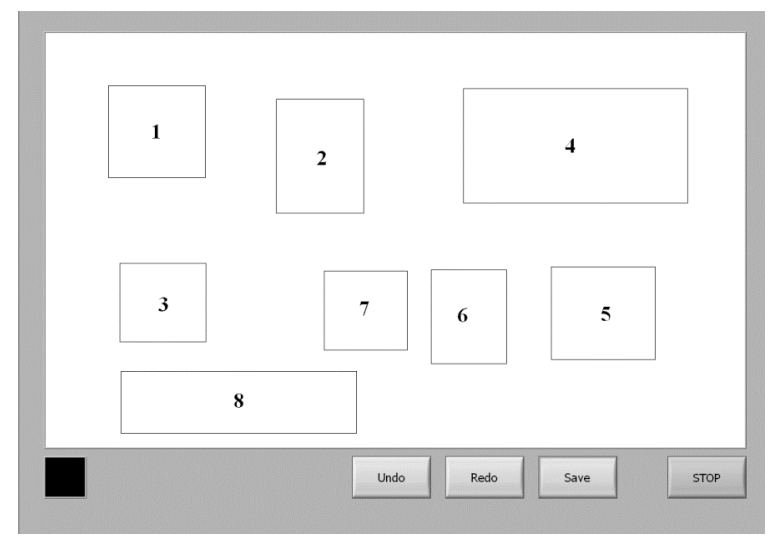

Figure 8. Layout generating using Template Generator.

A eight-element-layout is generated in which boxes are movable, deletable using functions provided by Template Generator (The number in each box is attached afterwards).

There are three formats of results generated by Template Generator, (1) TXT file, storing template data (coordinates of left-top and right-bottom points of the boxes); (2) JPG file, working as an illustration of the boxes and corresponded numbers; (3) XML (Extensible Markup Language) file, including the same information as TXT file, is an intermediate product which transfers the data from Template Generator to Data Recorder.

\section{Data Recorder}

The idea of 'Data Recorder' comes from the articles of Held (1999), Held et al. (1999) and Held \& Manser (2005), in which they use touch-screen to read in the coordinates of the typed points to simplify the traditional pen and paper data recording method. 'Data Recorder' is designed to import the layout created from Template Generator, record link events during the data recording process, and generate the final results with the assistance of the computer. The XML file generated from the Template Generator is read, as a pre-step before the data recording. Each event is registered by either using a mouse or a touch pen to select the corresponding boxes. When a box is selected, the number (name) of the box, coordinates and time stamp are recorded and stored into the memory of the computer. As illustrated in Figure 9, the different colours work as signals to indicate which two boxes have been selected previously. The red box was the last selected, while the green box was selected before the red one. The default colour is white and this shows a box was not selected for the last two steps/clicks. This colour changing process can also be treated as an indicator to check whether the box has been selected successfully.

In order to illustrate the versatility of the system, it is assumed that the sequence of elements operated during a task is '1-3-2-4-7-6-5-8-1-7-4-6-5-2'. Users record the link events by clicking inside each box according to the sequence. 'Save result' button enables the users to save the sequence and time stamp into the TXT file. Figure 10 indicates the link diagram result generated by 'Draw \& Save'.

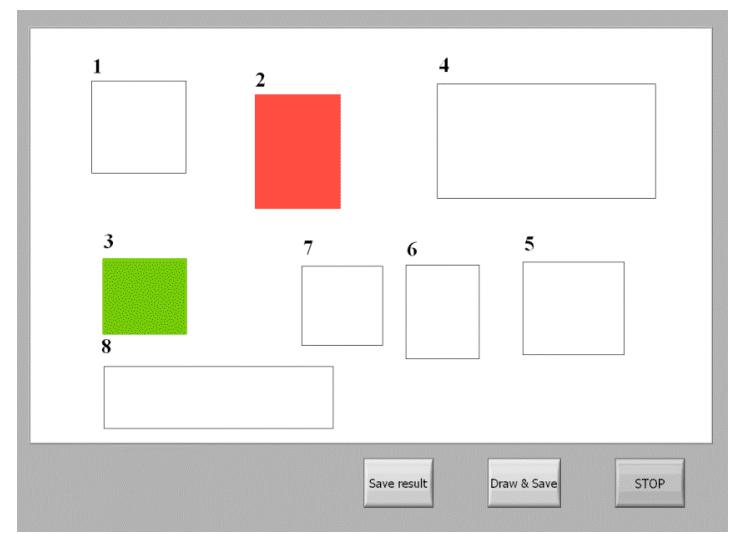


Figure 9. Event recording using Data Recorder.

Events are recorded by typing inside corresponding boxes, including coordinates of touched points and elapsed time (The number in each box is attached afterwards).

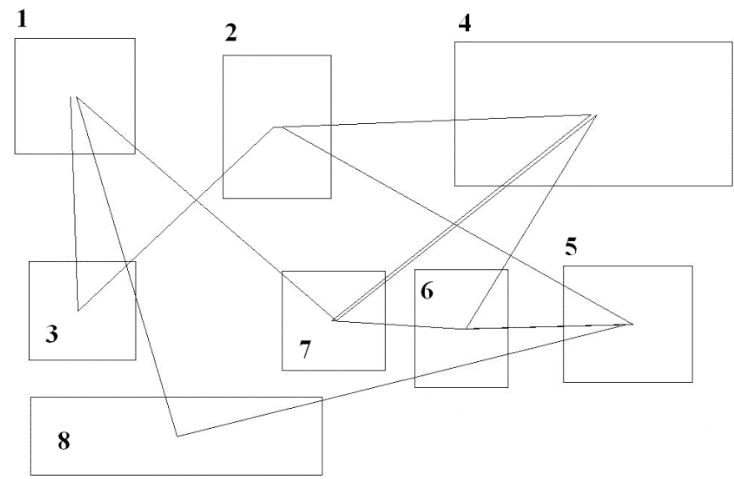

Figure 10. Link Diagram (The number in each box is attached afterwards).

\section{Further work}

The CLA system is at an early prototype stage, and much more work and information will be required to develop and test to enhance usability. Further work will include:

Template Generator

A option of entering the name for each box, rather than just numbering it; an option for reconstructing each box by modifying the data directly (coordinates); an option of inserting available templates (e.g. the template of a stretcher with a patient lying on it, which is frequently used when creating inner ambulance layout); a solution for drawing overlapping boxes; an option of sketching circles and triangles, is required.

\section{Data Recorder}

An option of indicating the direction of each link in the result; an option for producing Time-Event Diagram as a alternative result and outputting Event-Time List into Excel; a solution of selecting overlap boxes, needs to be added.

\section{System tests and validation}

As stated in 'Aims and objectives', several tests needs to be carried out for system validation of efficiency, accuracy, practicability with a range of users and real-time activities.

\section{CONCLUSION}

This paper discussed LA as both a layout analysing and a data recording method. The development of LA from a pen and paper method to computer-based system is presented as well as the differences in performing LA between the healthcare domain and other domains; limitations of the LA method; and current work with computers. Aims and objectives for the new system are summarized. Thus, Computerised Link Analysis, which enhances the performance and increases the efficiency of traditional LA, is being developed. The description of the system at an early prototype stage is presented to illustrate the versatility. Further work such as validation of CLA are also described.

\section{REFERENCES}

Beach, L., Chi, M., Klein, G., Smith, P. \& Vicente, K. (1997) Naturalistic decision making and related research lines, In Zsambok, C. E. \& Klein, G. A. (Ed.), Naturalistic decision making, New York: Lawrence Erlbaum Associates.

Beach, L. \& Mitchell, T. (1987) Image theory: Principles, goals, and plans in decision making, Acta Psychologica, $66(3), 201-220$.

Chapanis, A. (1959) Research techniques in human engineering, Baltimore: Johns Hopkins Press.

Endsley, M., Klein, G., Woods, D., Smith, P. \& Selcon, S. (1995) Future directions in cognitive engineering and naturalistic decision making, Proceedings of the Human Factors and Ergonomics Society 39th Annual Meeting, 913 October 1995, San Diego, California, 450-452.

Ferreira, J. \& Hignett, S. (2005) Reviewing ambulance design for clinical efficiency and paramedic safety, Applied Ergonomics, 36, 97-105.

Fitts, P.M., Jones, R.E. \& Milton, J.L. (1950) Eye movements of aircraft pilots during instrument landing approaches, Aeronaut. Eng. Rev., 9(2), 24-29.

Gilbreth, F. B. \& Gilbreth, L. M. (1917) Applied motion study, New York: The MacMillan Company. 
Glass, J. T., Zaloom, V. \& Gates, D. (1991) Computer-aided link analysis (CALA), Computers in Industry, 16(2), 179187.

Harper, W. \& Harris, D. (1975) The application of link analysis to police intelligence, Human Factors, 17(2), $157-164$.

Haygood, R. C., Teel, K. S. \& Greening, C. P. (1964) Link analysis by computer. Human Factors, 6, 63-70.

Held, J. (1999) Fitting the mental model: A new technique for computerised event recording, In Harris D. (Ed.), Engineering psychology and cognitive ergonomics, London: Ashgate.

Held, J., Bruesch, M., Krueger, H. \& Pasch, T. (1999) The FIT-system: A new hand-held computer tool for ergonomic assessment, Medical \& Biological Engineering \& Computing, 37(Supl 2), 862-863.

Held, J. \& Manser, T. (2005) A PDA-based system for online recording and analysis of concurrent events in complex behavioral processes, Behavior Research Methods, Instruments \& Computers, 37(1), 155-164.

Hignett, S. \& Lu, J. (2007) Evaluation of Critical Care Space Requirements for Three Frequent and High-Risk Tasks, Critical Care Nursing Clinics of North America, 19, 167-173.

Jones, A., Hignett, S. \& Benger, J. (2008) Identifying current and future care activities in ambulances, emergency departments and primary care, Emergency Medicine Journal, 25 (Suppl):A3

Klein, G. (1995) Naturalistic decision making, Proceedings of the Human Factors and Ergonomics Society 39th Annual Meeting, 9-13 October 1995, San Diego, California, 452-452.

Klein, G. (1997) An overview of naturalistic decision making applications, In Zsambok, C. E. \& Klein, G. A. (Ed.), Naturalistic decision making, New York: Lawrence Erlbaum Associates.

Klein, G. A. (1989) Recognition-primed decisions, In Rouse W. B. (Ed.), Advances in Man-Machine System Research, $5,47-92$.

Lippert, S. (1971) Travel in nursing units, Human Factors, 13(3), 269-282.

McCormick, E. J. (1970) Human factors engineering, New York: McGraw-Hill Education.

Mosier, K. L. \& Orasanu, J. (1995) Naturalistic Decision Making: A Domain-Integrated Overview, Proceedings of the Human Factors and Ergonomics Society 39th Annual Meeting, 9-13 October 1995, San Diego, California, $474-477$.

Nemeth, C. (2003) Human factors methods for design, Boca Raton: CRC Press.

Noble, D. (1989) Application of a theory of cognition to situation assessment, Vienna, VA: Engineering Research Associates.

Stuster, J. \& Chovil, A. (July, 1994) Tracking disease outbreak, Ergonomics in Design: The Quarterly of Human Factors Applications, 2(3), 8-15.

Thomson, R. M. (1972) Design of multi-Man-Machine work areas, In VanCott H.P. \& Kinkade R. G. (Ed.), Human engineering guide to equipment design, Washington, D.C.: US Government Printing Office.

Thorstensson, M., Axelsson, M., Morin, M. \& Jenvald, J. (2001) Monitoring and analysis of command post communication in rescue operations, Safety Science, 39(1-2), 51-60.

Zsambok, C. E. \& Klein, G. A. (1997), Naturalistic decision making, New York: Lawrence Erlbaum Associates. 